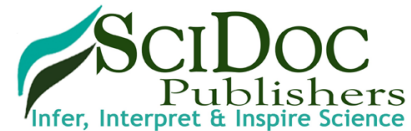

International Journal of Vaccines and Research (IJVR)

ISSN 2572-7427

\title{
Vaccine killed Kanchha'(?): an analysis of pros vs cons views
}

Review Article

Ghimire TR

Faculty of Science, Nepal Academy of Science and Technology (NAST), Lalitpur, Nepal.

\section{Abstract}

Though vaccines are the most successful interventions of the biomedical sciences, few people have negative aspects of vaccination. Due to greater demands of vaccines against HIV, malaria, and TB, vaccinologists are in the hope of current research for the newer vaccines. This viewpoint addresses the current opinions of provaccinists and antivaccinists in an area where people do not know much about vaccination. This paper concludes that vaccination is one of the boons of biomedical sciences and it needs an extra care during preservation, transportation and preparation of vaccines in injection. This will be supportive to successfully immunize the children and to lower the negative perception of antivaccinists.

Keywords: Cold Chain; Live Vaccines; Measles; Safety and Economic Issue.

Abbreviations: BCG: Bacillus Calmette-Guerin; TB: Tuberculosis; HIV: Human Immunodeficiency Virus; EPI: Expanded Program on Immunization; MMR: Measles, Mumps, and Rubella; EPS: Efficacy, Potency, and Stability.

\section{Introduction}

It is a widely believed fact that vaccines are the most successful interventions of the biomedical sciences. The implication of vaccines have decreased the mortality of morbidity of children from various diseases, however there are few people in developing countries who believe that vaccines may not be right choices of disease prevention. This paper contributes to the discussion of the potential perception regarding antivaccination and provaccination among people of different socio-educational status.

When I was having a break after working in the Immunology Laboratory of the University of Glasgow, Scotland, I read a news from online source that Kanchha in Nepal died after few hours he was given Measles immunization. Kanchha was from a middleclass family of Nepal. He was born after 5 years of his parents' marriage. During these periods, Kanchha's grandma had taken his mum to various witchdoctors and pundits. She used to take fasting once a week. His grandpa had organized numerous ritual activities. Moreover his dad took her to the Ayurvedic hospitals. Even his maternal uncle made her accessible to various medical hospitals in Kathmandu. All their unified endeavors were to concentrate on having a baby from her. I am not sure whether these means helped or not, but Kanchha was born. Everyone was happy in the family due to the presence of a newly born cute baby.

When Kanchha's parents were trying to take him to hospital for Bacillus Calmette-Guerin (BCG) vaccination, his grandma said to them 'I don't think your immunization will prevent tuberculosis (TB) by BCG vaccination. To prevent TB, keep on performing 'Ancestral Worship'.

'Mum! You are just a conservative woman!' Kanchha's dad opposed to her at once.

'No, we don't need to vaccinate a baby. If you feed them well with lots of fruits and nutrients, you can protect your baby'. Mum tried to convince him.

'Mum, you are just joking! We need to vaccinate a baby to prevent the future infection'. Kanchha's dad quickly responded to her.

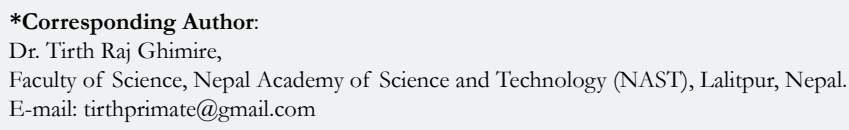

Copyright: Ghimire TR ${ }^{\circ}$ 2016. This is an open-access article distributed under the terms of the Creative Commons Attribution License, which permits unrestricted use, distribution and reproduction in any medium, provided the original author and source are credited.

†A fictional Nepalese child known to me from reading news of the Nepalese community. I am not responsible for any similarity to an actual person with Kanchha. If this happens, it will be unintentional and purely coincidental. The term has been changed to Kanchhas to indicate its plural form. This name has been written to express a love to a baby and must not be read as an indication of any partiality in the context of baby's color, age, or status. 
Your brain is just malfunctioned due to so-called vaccine books! Do you have any scientific proof that vaccine works?' Mum showed her doubtfulness regarding the effectiveness of vaccines.

Yes, I have. The human smallpox infection and some animal infections such as swine cholera, parvovirus-induced enteritis, distemper virus, and pseudo-rabies virus have been controlled and prevented by employing vaccination [1]. The worldwide campaign towards the prevention of polio is also under the road of success $[2,3]$. There are many proofs that vaccines reduce the morbidity and mortality of children from infectious diseases. For example, let me describe about the mortality due to Measles that has been halved in five years to 500,000 per annum. The basic global vaccination program, Expanded Program on Immunization (EPI), has prevented about 1 million deaths per annum' [4]. Kanchha's dad tried to justify that vaccination works.

You are being a scientist with just a shit of a blind faith! Vaccine kills the healthy people. Vaccination means you are supplying microorganisms in your body! If you don't believe, ask your dearest scientists!' Mum cried.

'Oh God! Vaccines never kill people! I know 21 dollars are saved for every dollar spent on the Measles, mumps and Rubella (MMR) vaccine [5]. So, vaccines are one of the most economic and safest interventions of biomedical research. I don't know why my mum is being so rude to vaccination' Kanchha's dad murmured.

But he didn't try to defend his mum because he had already known that his mum was antivaccinist. So, he just laughed at her, ignored her views and went to the government hospital to vaccinate Kanchha by BCG. They took Kanchha at exactly the same day that nurses and midwives had invited on. Kanchha suffered from normal reactions such as mild fever and inflammation at the site of immunization following vaccination.

Kanchha became nine-month-old. His teeth were about to erupt. He was cute, smiling and was perfectly healthy. His family used to feed him cow's milk, home-made Sarbottam $\mathrm{Lito}^{2}$, fruits containing banana, pomegranate, apple, and orange. His grandma started to feed him various domestic herbs which she believed he does not need any medications such as vaccines and drugs in future. In contrast to her ideologies, Kanchha's father took him to the central district hospital for immunization with Measles vaccine. He was very happy supposing that he was going to protect Kanchha from Measles. Unfortunately, Kanchha became suffered from extreme fever, diarrhea, and sweating after about 8 hours of immunization. He became pale and immediately died. It was an irreversible loss for Kanchha's family.

\section{Discussion}

This story arose several questions in mind. I cannot say who created Kanchha, God or Nature? And who killed Kanchha? God or Nature? To some extent, our negligence might be the cause of his death. To understand this, we should discuss the views of Kanchha's father and his grandma.

Firstly, I would focus on his father's positive views of vaccination. He is absolutely right that babies should be vaccinated to prevent from the vaccine preventable diseases such as diphtheria, Haemophilus influenzae type $\mathrm{b}$ infection, hepatitis $\mathrm{B}$ viral infection, tetanus, Measles, mumps, neonatal tetanus, pertussis, pneumococcal infection, rubella, and serogroup $\mathrm{C}$ meningococcal infection [3-6]. He was also true that vaccines, without any doubt, are the most effective tools of biomedical sciences.

Let's discuss the views of Kanchha's grandma about vaccines. She

Figure 1. A cons vs pros ideology regarding vaccine and vaccination. An individual who is against vaccines have several self assumptions. A provaccinist has scientific evidences and believes on science. $\mathrm{He} / \mathrm{she}$ is optimistic to the discovery of future vaccines against major killers such as TB, HIV, flu, and malaria.

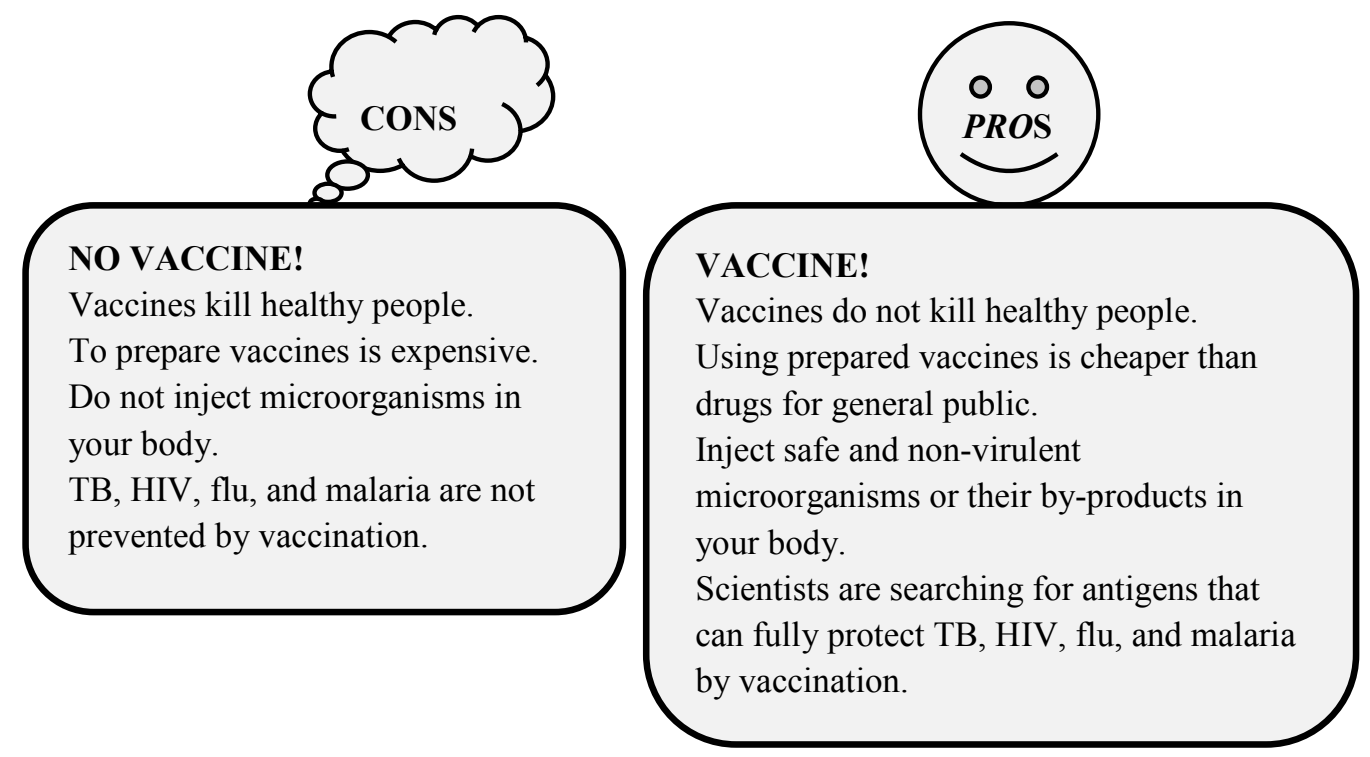

1. A worship or ritual activity by the group of people of similar castes and surnames.

2. Flour porridge or Super Flour, it is a home-processed weaning food in Nepal. Please visit the website about this: http://www.cf-hst.net/unicef-temp/Doc-Repository/doc/doc481799.PDF (Accessed on 01 August 2015). 
is partly right that vaccination means the supply of microbes in the host body. To elaborate these issues, let's know the terms that are dealt under vaccinology. Normally, the terms vaccine, vaccination and immunization have been synonymously used in nonmedical parlance. However, with better understanding of principles and techniques of immunology, their definitions have been more precise in biomedicine. Vaccination and immunization denote the method of inoculating vaccines to the body of the hosts. Vaccines are the liquid suspension of the live attenuated or killed microorganisms, or their part or by-products which can produce the protective immune responses when inoculated in the host body. By using these principles, scientists have discovered several vaccines such as killed vaccines, live attenuated vaccines, toxoid subunit vaccines, and conjugate vaccines. Among these vaccines, I will explain about live vaccines and their process of development which is partly matched with the views of Kanchha's grandma.

Live vaccines, for instance, vaccines against Measles, mumps, and chickenpox contain modified live microorganisms. The modified organisms contain reduced virulence made by either multiple passages in embryonated eggs or tissue culture cells by chemical mutagenesis or under suboptimal conditions of temperature [7, 8]. These modified organisms replicate in the host, causing a mild infection and consequently stimulate the protective host immune response sufficiently in a manner similar to the immune response generated by natural infection.

One important point why Kanchha's grandma became discontented for vaccination might be due to the spending of large sum of money invested on vaccine development (Figure 1). She might have believed that the money spent could be utilized for food or fruits or for herbs that could protect babies from infectious diseases. Vaccine development is a slow process. It includes identification of particular antigens, its in vitro and in vivo effects on mammalian cells and the consequent clinical trials in humans. As a result, expanding the time and size of vaccine clinical trials may lead to the delayed adverse effects. Then, it results in the high expenditure of private and public donors and shareholders. Thus, vaccinology has been increasingly shaped by the rules and regulations of biomedical and social ethics, safety standards and financial constraints. In summary, it is very difficult to unequivocally support the quality and the safety of pre- and post-licensed vaccines individually as well as publicly.

Grandma's views about BCG vaccination are mostly right. This is because scientists are formulating several hypotheses and actively working on why BCG is not fully functioning on humans. BCG is inefficient against reactivation or reinfection of Mycobacterium spp though it is effective against tuberculous meningitis. Notably, the protective efficacy against adult pulmonary TB ranges from $-35 \%$ to $+80 \%$ depending on different populations of various geographic areas [9-11]. Kanchha's Grandma might have several years of experiences on listening the news that broadcasts more than 40,000 new cases of TB per annum resulting in the annual death of about 5,000 - 7,000 thousand TB patients; however BCG is actively employed in Nepal [12].

Let us concentrate on Kanchha's death. We knew that he was immunized before he died. But even a layman acknowledges that Measles vaccines are supposed to be one of the most efficient vaccines. These vaccines are given subcutaneously to children after the age of 9 month [13]. Measles vaccines are light sensitive.
So, these vaccines are supplied in amber-colored vials. They are also heat sensitive before and after reconstitution. So, the damage caused by heat is cumulative and cannot be reversed by re-freezing these vaccines. Once the Measles vaccines are reconstituted, there is risk of contamination because unfortunately, these vaccines do not contain preservatives. That is why they should not be used after 4 hours of reconstitution [13].

Notably, Measles vaccines are supplied in a cold chain system that secures the major factors of vaccines such as efficacy, potency, and stability (EPS). Cold chain is a process of maintenance of the constant temperature (maximum $+8^{\circ} \mathrm{C}$ to minimum $-20^{\circ} \mathrm{C}$ ) of Measles vaccines during their storage and transportation from the place of manufacture to the spot of immunization [14]. Here, the 'spot' means the place where children are immunized. However, due to the recklessness of health workers or store-keepers, or technical problems of freezers, or refrigerators, or coolers, or any other instruments, the cold chain system may be broken and the EPS may be destroyed. So, I think, Kanchha might have died owing to defective cold chain system and the consequent contamination of Measles vaccines that might have been used long after 4 hours of reconstitution. I, therefore, strongly believe that vaccines never kill humans but extreme carelessness during preservation, their transportation and preparation in injection may kill these nature's most beautiful and civilized species. If we are careful about the blunder that may happen during its preservation and transportation, we can save the lives of many Kanchhas of our society in future. This can also make it easier to erase the false conviction of antivaccinists.

Undoubtedly speaking, children are the pillars of the nation. The young ones of today are likely to run the country tomorrow. I am really grateful to the UNICEF for the statement about the significance of child health [15]. This will be applicable not only for the affluent countries but also for the disadvantaged nations like Nepal.

"We strive towards the day when nations will be judged not by their military or economic strength, nor by the splendor of their capital cities andpublic buildings, but by the well-being of their children [15]".

\section{Conclusions}

While biomedical sciences are the boon of the modern age, few people in disadvantage nations think that these sciences are not necessary to upgrade the health of a baby. This impression has resulted in the failure of the immunization success in these countries. In addition, the minor mistake of health workers during handling of vaccines before immunization may lead to death of immunized individuals. Thus, an extra care should be taken during preservation, transportation and preparation of vaccines in injection. This will be supportive to successfully immunize the children and to lower the negative perception of antivaccinists.

\section{Conflict of Interest Statement}

I am a provaccinist. The views expressed here may not necessarily represent the official views of Nepal Academy of Science and Technology (NAST), Lalitpur, Nepal. I declare that I do not have any competing interests. 


\section{Acknowledgements}

My special acknowledgement goes to all those Kanchhas who died on post-vaccination period. No external funding was received for the production of this paper.

\section{References}

[1]. Wilson-Welder JH, Torres MP, Kipper MJ, Mallapragada SK, Wannemuehler MJ, et al. (2009) Vaccine adjuvants: current challenges and future approaches. J Pharm Sci 98(4): 1278-1316.

[2]. WHO (2010) Polio vaccines and polio immunization in the pre-eradication era: WHO position paper. Wkly Epidemiol Rec 85(23): 213-228.

[3]. WHO (2010) WHO vaccine-preventable diseases:monitoring system, 2010 global summary. In. Edited by The Department of Immunisation VaB: World Health Organisation. 1-31.

[4]. Miller MA, Sentz JT (2011) Vaccine-Preventable Diseases.

[5]. Anonymous (2008) Understanding vaccines what they are how they work. In. US Department of Health and Human Services National Institute of Health National Institute of Allergy and Infectious Diseases: US Department of Health and Human Services National Institute of Health National Institute of Allergy and Infectious Diseases.

[6]. Rappuoli R, Miller HI, Falkow S (2010) The Intangible Value of Vaccination. Science 297(5583): 937-939.
[7]. Bonanni P, Santos, JI (2011) Vaccine evolution. Perspectives in Vaccinology 1(1): 1-24.

[8]. Ebensen T, Link C, Guzman CA, Stefen HEK (2004) Classical Bacterial Vaccines: In: Novel vaccination strategies. WILEY-VCH Verlag GmbH \& Co. KGaA, Weinheim. 221-240.

[9]. Fine PE (1989) The BCG story: lessons from the past and implications for the future. Rev Infect Dis 11(Suppl 2): S353-359.

[10]. Colditz GA, Brewer TF, Berkey CS, Wilson ME, Burdick E, et al. (1994) Efficacy of BCG vaccine in the prevention of tuberculosis. Meta-analysis of the published literature. JAMA 271(9): 698-702.

[11]. Roche PW, Triccas JA, Winter N (1995) BCG vaccination against tuberculosis: past disappointments and future hopes. Trends Microbiol 3(10): 397-401.

[12]. NTC (2013) TB Burden in Nepal. In 2013 edtn: Government of Nepal, Ministry of Health and Population, Department of Health Services, National Tuberculosis Center.

[13]. Anonymous (2010) Handbook for Vaccine \& Cold Chain Handlers. In. Department of Health \& Family Welfare Ministry of Health and Family Welfare Government of India.

[14]. WHO (1999) Temperature monitors for vaccines and the cold chain. Department of vaccines and other biologicals. CH-1211 Geneva 27, Switzerland. 1-23.

[15]. UNICEF (2004) Progress for Children. In. Division of Communication, 3 United Nations Plaza, H-9F New York, NY 10017, USA. 